\title{
Synthesis and Biological Evaluation of Novel Water-Soluble Poly-(ethylene glycol)-10-hydroxycamptothecin Conjugates
}

\section{Na Guo, Du Jiang, Luyao Wang, Xing You, Yu-Ou Teng and Peng Yu *}

Key Laboratory of Industrial Microbiology, Ministry of Education, Tianjin Key Laboratory of Industry Microbiology, College of Biotechnology, Tianjin University of Science and Technology, No.29, 13th Avenue, TEDA, Tianjin 300457, China; E-Mails: guona@tust.edu.cn (N.G.); jiangduwill@126.com (D.J.); wangluyao_1106@163.com (L.W.); youxing_2013@126.com (X.Y.); tyo201485@tust.edu.cn (Y.-O.T.)

* Author to whom correspondence should be addressed; E-Mail: yupeng@tust.edu.cn; Tel.: +86-22-6091-2562; Fax: +86-22-6060-2298.

Academic Editor: Jean Jacques Vanden Eynde

Received: 20 April 2015 / Accepted: 15 May 2015 / Published: 21 May 2015

\begin{abstract}
In order to improve the antitumor activity and water solubility of 10-hydroxycamptothecin (HCPT), a series of novel HCPT conjugates were designed and synthesized by conjugating polyethylene glycol (PEG) to the 10-hydroxyl group of HCPT via a valine spacer. The in vitro stability of these synthesized compounds was determined in pH 7.4 buffer at $37{ }^{\circ} \mathrm{C}$, and the results showed that they released HCPT at different rates. All the compounds demonstrated significant antitumor activity in vitro against K562, HepG2 and HT-29 cells. Among them, compounds, 4a, 4d, 4e and 4f, exhibited 2-5 times higher potency than HCPT. The stability and antitumor activity of these conjugates were found to be closely related to the length of PEG and the linker type, conjugates with a relatively short PEG chain and carbamate linkages (compounds $\mathbf{4 a}$ and $\mathbf{4 f}$ ) exhibited controlled release of HCPT and excellent antitumor in vitro activity.
\end{abstract}

Keywords: antitumor; PEG; hydroxycamptothecin; conjugate 


\section{Introduction}

Camptothecin (CPT) and 10-hydroxycamptothecin (HCPT) are natural occurred topoisomerase I inhibitors with strong antitumor activity both in vitro and in vivo [1,2]. However, the clinical use of these two compounds is limited by their low solubility in water and high toxicity. In recent years, the potential of synthetic macromolecules as carriers of anticancer drugs has attracted immense interest. Polymeric prodrugs of various cytotoxic agents with soluble polymers such as PEG have been extensively investigated. The polymeric conjugates could simultaneously act as both a transport form and a prodrug to enhance drug distribution in tumor sites by enhanced permeability and retention (EPR) effects and improved drug pharmacokinetic properties [3-6]. In the past few years, CPT has been covalently coupled at C-20 to various water-soluble polymers to improve its solubility [7], i.e., PEG, poly(L-glutamic acid), $\beta$-cyclodextrin-based polymers, poly[ $N$-(2-hydroxypropyl)-methacrylamide], poly[( $N$-carboxybutyl)-L-aspartamide] and poly(amido-amine).

PEG, a water-soluble, nontoxic, biodegradable and biocompatible polymer, is broadly used in medicine, food and cosmetics. Plenty of PEG-CPT conjugates have been reported, especially with an ester linkage [8]. However, PEG has seldom been applied for the synthesis of HCPT-PEG conjugates, especially with linkages other than ester ones, although HCPT is more active and less toxic than CPT. Derivation at C-10 of CPT analogues has led to some successful prodrugs, for example, irinotecan (Figure 1), which is a water-soluble prodrug of the CPT derivative SN-38 with a carbamate linkage. It is the most widely used CPT derivative for the treatment of cancer. However, the low bioconversion efficiency $(4 \%-5 \%)$ from irinotecan to the active form $\mathrm{SN}-38$ is responsible for individual variation in efficacy and toxicity [9-11].
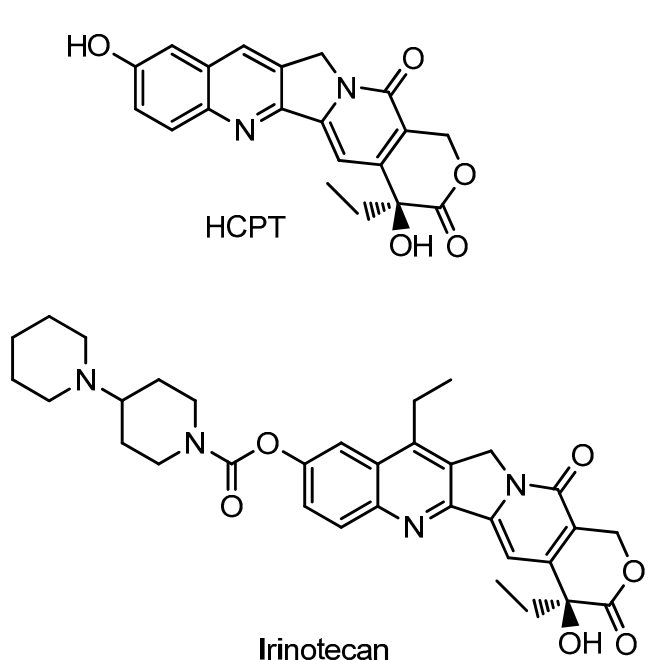
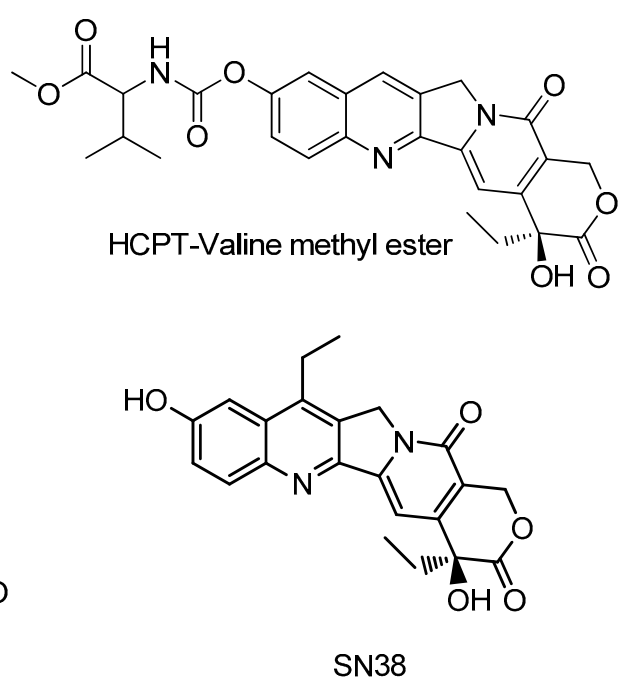

Figure 1. Structures of HCPT, HCPT-valine methyl ester, irinotecan and SN38.

In our previous study [12], a series of amino acid methyl ester conjugates of HCPT at C-10 were found to exhibit excellent antitumor activity and decreased toxicity against several normal cells, especially the valine methyl ester conjugated derivative (HCPT-Valine methyl ester, Figure 1). Here, we would like to report the design and synthesis of a series of PEG-HCPT conjugates based on this 
valine spacer. Our results demonstrated that the HCPT release and the antitumor activity are strongly dependent on the PEG length and the linker properties.

\section{Results and Discussion}

In order to conjugate PEG with HCPT, valine was chosen as a spacer to link the PEG and HCPT (compounds 4a-4f). Meanwhile, PEG and HCPT were also conjugated directly via a carbonate group (compound 5) or an ether group (compound 6) as reference compounds to investigate the influence of the spacer on stability and activity (Figure 2).

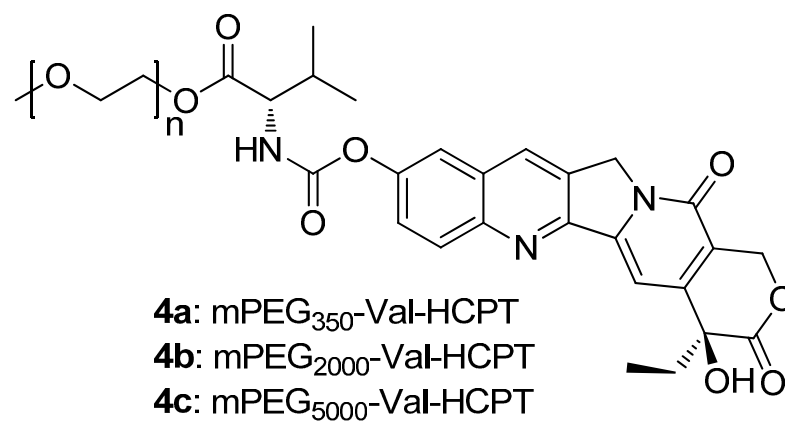

4c: $\mathrm{mPEG}_{5000}-\mathrm{Val}-\mathrm{HCPT}$<smiles>CC[C@]1(O)C(=O)OCc2c1cc1n(c2=O)-c2cc3ccccc3nc2C1</smiles>

4d: HCPT-Val-PEG $2000-$ Val-HCPT

4e: HCPT-Val-PEG $20000^{-V a l-H C P T}$

4f: HCPT-Val-PEG 300 -Val-HCPT<smiles>[2H]NC(=O)O</smiles><smiles>CC(C)[C@H](C)C(=O)OCCOC(=O)[C@@H](NCO)C(C)C</smiles>

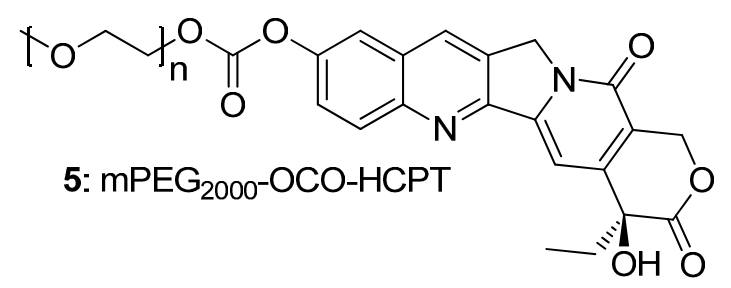

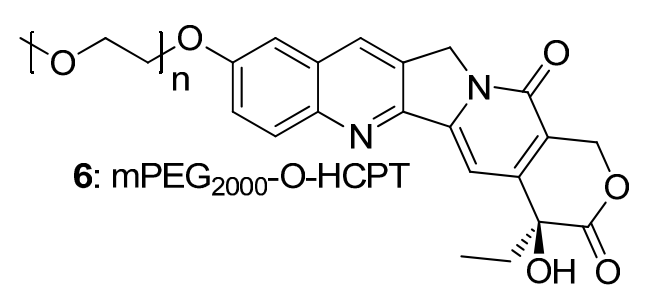<smiles>CC[C@@]1(O)C(=O)OCc2c1cc1n(c2=O)Cc2cc3cc(OC(N)=O)ccc3nc2-1</smiles>

Figure 2. Structures of conjugates $\mathbf{4 a - 4 f , 5}$, and $\mathbf{6}$.

\subsection{Chemistry}

The synthesis of the conjugates $\mathbf{4 a}-\mathbf{4 f}$ is shown in Scheme 1. The key intermediate 1a was synthesized in a good yield from HCPT and 4-nitrophenyl chloroformate in the presence of triethylamine. Meanwhile, Boc-valine was linked with monomethoxy-PEG (mPEG) or dihydroxy-PEG via an ester group to afford mPEG-valine-Boc (compounds 2a-2c) or Boc-valine-PEG-valine-Boc (componds $\mathbf{2 d - 2 f}$ ), which were then deprotected with TFA and reacted with 1a to provide the coupled compounds $\mathbf{4 a}-\mathbf{4 f}$.

Compound 5 (Scheme 2) was synthesized using a similar method to that described for compounds 4a-4f. $\mathrm{MPEG}_{2000}$ was reacted with 4-nitrophenyl chloroformate to give the active intermediate $\mathbf{1 b}$, which was then reacted with HCPT to afford $\mathrm{mPEG}_{2000}-\mathrm{OCO}-\mathrm{HCPT}$ (5). The synthesis of $\mathrm{mPEG}_{2000}-\mathrm{O}-\mathrm{HCPT}(\mathbf{6})$ is shown in Scheme 3. MPEG $_{2000}$ was converted into compound 8 via $p$-toluenesulfonate 7. HCPT was reacted with 8 under basic conditions to afford compound 6 (Scheme 3). 
<smiles>CC[C@]1(O)C(=O)OCc2c1cc1n(c2=O)Cc2cc3cc(O)ccc3nc2-1</smiles>
HCPT

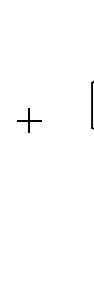<smiles>O=C(Cl)Oc1ccc([N+](=O)[O-])cc1</smiles>
CI<smiles>CO[C@@]1(O)C(=O)OCc2c1cc1n(c2=O)Cc2cc3cc(OC(=O)Oc4ccc([N+](=O)[O-])cc4)ccc3nc2-1</smiles>

$1 a$
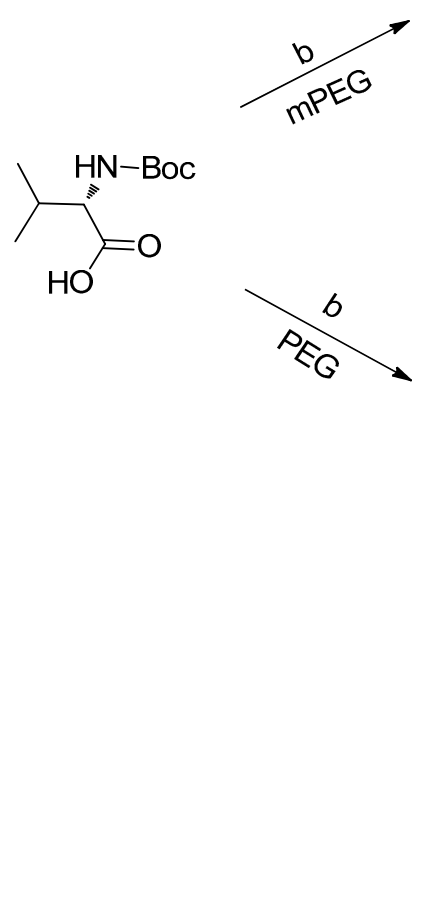

$1 a+3 a-3 f \longrightarrow d$

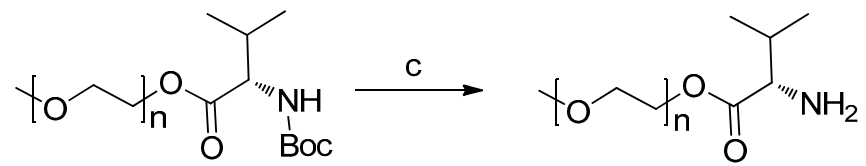

2a: $\mathrm{mPEG}_{350}$-Val(Boc)

2b: $\mathrm{mPEG}_{2000}-\mathrm{Val}(\mathrm{Boc})$

2c: $\mathrm{mPEG}_{5000}-\mathrm{Val}(\mathrm{Boc})$ 3a: $\mathrm{mPEG}_{350}$-Val

3b: $\mathrm{mPEG}_{2000}-\mathrm{Val}$ 3c: $\mathrm{mPEG}_{5000}$-Val

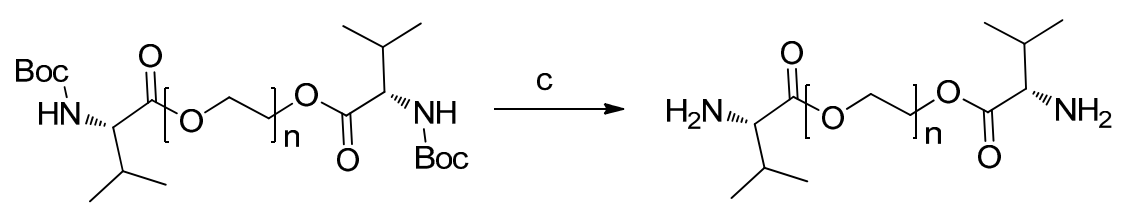

2d: $\operatorname{Val}(\mathrm{Boc})-\mathrm{PEG}_{2000}-\mathrm{Val}(\mathrm{Boc})$

2e: $\mathrm{Val}(\mathrm{Boc})-\mathrm{PEG}_{20000}-\mathrm{Val}(\mathrm{Boc})$

2f: Val(Boc)-PEG 300 -Val(Boc) 3d: Val-PEG $2000-$ Val

3e: Val-PEG 20000 -Val

3f: Val-PEG $300-$ Val<smiles>CC(C)[C@H](NC(=O)Oc1ccc2nc3c(cc2c1)Cn1c-3cc2c(c1=O)COC(=O)[C@]2(C)O)C(=O)O[Ga]COC(C)(C)C</smiles>

4a: $\mathrm{mPEG}_{350}-$ Val-HCPT
4b: $\mathrm{mPEG}_{2000}-$ Val-HCPT
4c: $\mathrm{mPEG}_{5000}-$ Val-HCPT

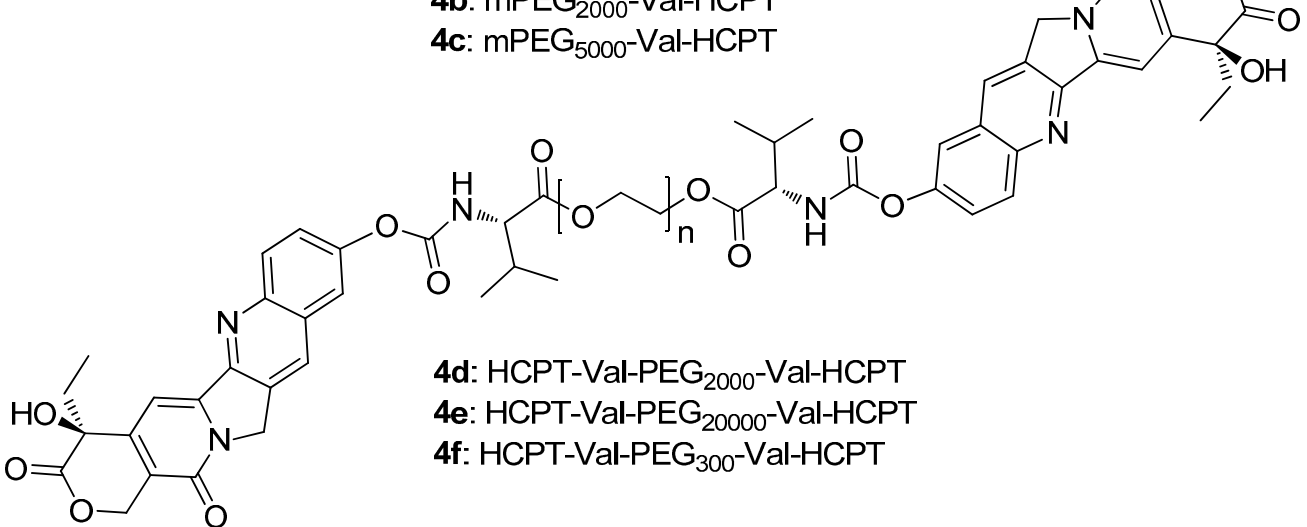

Reagents and conditions: (a) Et 3 N, THF, rt. 1 h; (b) EDCI, DMAP, rt. overnight; (c) TFA, DCM, rt. 1 h; (d) DMF, DCM, rt. 1 h.

Scheme 1. Synthesis of conjugates $4 a-4 f$. 


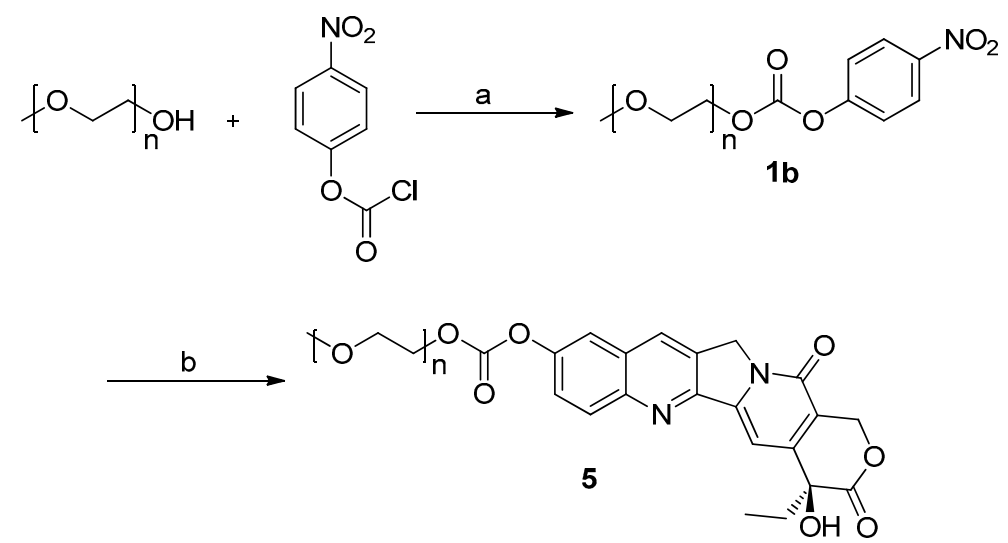

Reagents and conditions: (a) Et 3 N, DCM, rt. 3 h; (b) HCPT, Et ${ }_{3} \mathrm{~N}, \mathrm{DMF}, 60{ }^{\circ} \mathrm{C}, 45 \mathrm{~min}$.

Scheme 2. Synthesis of conjugate 5.

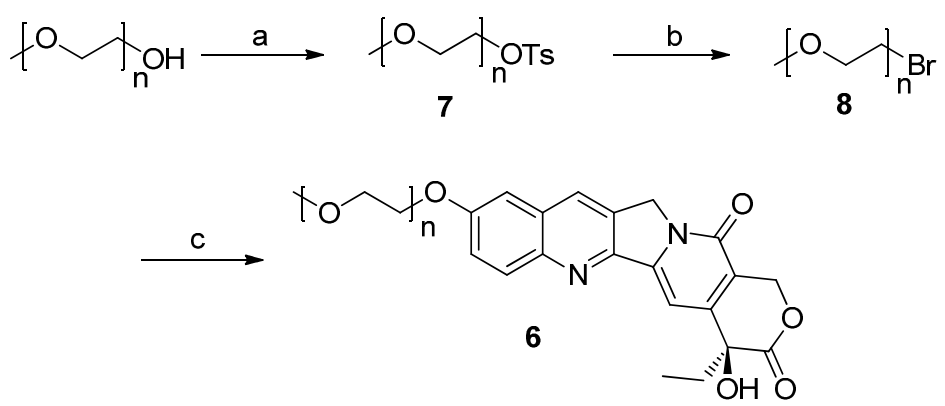

Reagents and conditions: (a) TsCl, Et ${ }_{3} \mathrm{~N}, \mathrm{DCM}$, reflux, 5 h; (b) LiBr, acetone, reflux, overnight; (c) HCPT, $\mathrm{K}_{2} \mathrm{CO}_{3}, \mathrm{DMSO}, 60^{\circ} \mathrm{C}, 3 \mathrm{~h}$.

Scheme 3. Synthesis of conjugate 6.

\subsection{Cytotoxicity}

The in vitro antitumor activity was evaluated on the K562, HepG2 and HT-29 cancer cell lines by the MTT assay, using HCPT as the reference compound. As shown in Table 1, the conjugates containing valine spacers (compounds 4a-4f) exhibited comparable or more potent (2-5 times) antiproliferative activity than HCPT. The most potent monovalent compound was $\mathbf{4 a}$, which contained a short mPEG chain, while $\mathbf{4 b}$ and $\mathbf{4 c}$ possessed comparable activity to HCPT. The IC 50 values of $\mathbf{4 d - 4 f}$ were almost the same as that of $\mathbf{4 a}$. Compared to the valine-linked conjugates, the activity of compounds $\mathbf{5}$ and $\mathbf{6}$ were significantly reduced, especially for $\mathbf{6}$.

\subsection{Stability and Conversion}

The stability of these conjugates was studied by HPLC at pH 7.4 in Phosphate Buffered Saline (PBS) at $37^{\circ} \mathrm{C}$ (Table 2). The results are showed in Table 2, the conjugates' stability is closely related to the length of PEG and the liker type. For compounds $\mathbf{4 a - 4 f}$ with valine spacers, the short PEG linked conjugates (4a and $\mathbf{4 f}$ ) exhibited controlled release of HCPT. The monovalent conjugate $\mathbf{4 a}$ released HCPT at a slower rate than $\mathbf{4 b}-\mathbf{4 e}$. Surprisingly, the bivalent conjugate $\mathbf{4 f}$ released HCPT very slowly, even slower than compound $\mathbf{6}$ which possessed an ether bond. The difference in stability of 
conjugates with different PEG chain lengths via carbamates (compounds $4 \mathbf{a}-\mathbf{4 f}$ ) maybe resulted from their different conformations in solution.

Table 1. Antiproliferative activity of the conjugates in vitro.

\begin{tabular}{cccc}
\hline \multirow{2}{*}{ Compound } & \multicolumn{3}{c}{$\mathbf{I C}_{\mathbf{5 0}}(\boldsymbol{\mu M} \mathbf{M})$} \\
\cline { 2 - 4 } & $\mathbf{K 5 6 2}$ & $\mathbf{H e p G 2}$ & $\mathbf{H T}-29$ \\
\hline $\mathrm{HCPT}$ & $0.11 \pm 0.07$ & $0.08 \pm 0.02$ & $0.22 \pm 0.09$ \\
$\mathbf{4 a}$ & $0.02 \pm 0.01$ & $0.04 \pm 0.01$ & $0.04 \pm 0.01 *$ \\
$\mathbf{4 b}$ & $0.12 \pm 0.03$ & $0.11 \pm 0.06$ & $0.19 \pm 0.10$ \\
$\mathbf{4 c}$ & $0.06 \pm 0.03$ & $0.12 \pm 0.08$ & $0.21 \pm 0.10$ \\
$\mathbf{4 d}$ & $0.02 \pm 0.01$ & $0.04 \pm 0.02$ & $0.04 \pm 0.01 *$ \\
$\mathbf{4} \mathbf{4}$ & $0.03 \pm 0.01$ & $0.05 \pm 0.03 *$ & $0.14 \pm 0.09$ \\
$\mathbf{4 f}$ & $0.02 \pm 0.01$ & $0.05 \pm 0.01$ & $0.04 \pm 0.01 *$ \\
$\mathbf{5}$ & $0.24 \pm 0.05 *$ & $0.39 \pm 0.23$ & $0.42 \pm 0.06 *$ \\
$\mathbf{6}$ & $26.99 \pm 0.74 * *$ & $32.30 \pm 1.48 * *$ & $42.97 \pm 16.67 * *$ \\
\hline \multicolumn{4}{c}{$* p<0.05 ; * *<0.01$, compared with the control group $(\mathrm{HCPT})}$.
\end{tabular}

Table 2. Stability of the conjugates at $\mathrm{pH}$ 7.4.

\begin{tabular}{ccccc}
\hline \multirow{2}{*}{ Compound } & \multicolumn{4}{c}{ Released HCPT (\%) } \\
\cline { 2 - 5 } & $\mathbf{1 ~ h}$ & $\mathbf{2} \mathbf{~ h}$ & $\mathbf{3 ~ h}$ & $\mathbf{4} \mathbf{~ h}$ \\
\hline $\mathbf{4 a}$ & 75 & 80 & 82 & 82 \\
$\mathbf{4 b}$ & 96 & 100 & - & - \\
$\mathbf{4 c}$ & 100 & - & - & - \\
$\mathbf{4 d}$ & 70 & 90 & 94 & 96 \\
$\mathbf{4 e}$ & 100 & - & - & - \\
$\mathbf{4 f}$ & 20 & 32 & 35 & 38 \\
$\mathbf{5}$ & 54 & 65 & 68 & 68 \\
$\mathbf{6}$ & 33 & 45 & 54 & 56 \\
\hline
\end{tabular}

\subsection{Discussion}

In this study, a series of novel conjugates of HCPT were designed and synthesized using PEG as carrier. The water solubility of all the compounds was significantly improved by introducing the water-soluble PEG chain. Considering the stability and the antitumor activity together, it was supposed that the active form of monovalent compounds $\mathbf{4 b}$ and $\mathbf{4 c}$ was HCPT, since they released almost $100 \%$ of their HCPT in a very short time and exhibited comparable IC $_{50}$ values as HCPT. Similarly, bivalent $\mathbf{4 d}$ and $\mathbf{4 e}$ also released HCPT very fast, but their IC $_{50}$ values are lower than HCPT, which is probably mainly due to the bivalence effect. Compounds $\mathbf{5}$ and $\mathbf{6}$, showed reduced activity (especially $\mathbf{6}$ ), maybe because their HCPT release rates are significantly reduced and they didn't exhibited cytotoxity by themselves, while conjugates $\mathbf{4 a}$ and $\mathbf{4 f}$, linked with relatively short PEGs, showed excellent activity and controlled release of HCPT. The reason that conjugates $\mathbf{4 a}$ and $\mathbf{4 f}$ released HCPT more slowly is probably because their conformations differ from other conjugates containing longer PEG chains and valine spacers (compounds $\mathbf{4 b - 4 e}$ ), while they exhibited better activity than HCPT perhaps because $4 \mathbf{a}$ 
and $\mathbf{4 f}$ could directly interact with topoisomerase I by themselves too. Of course, this speculation needs to be further confirmed.

\section{Experimental Section}

\subsection{General Information}

${ }^{1} \mathrm{H}-\mathrm{NMR}$ spectra were recorded on a Bruker AM-400 NMR spectrometer (Zurich, Switzerland) in DMSO- $d_{6}$. The chemical shifts are reported in $\delta(\mathrm{ppm})$ relative to tetramethylsilane as the internal standard. The mass spectra were obtained on a Bruker MALDI-TOF mass spectrometer (Billerica, MA, USA). Thin-layer chromatography (TLC) was performed using E. Merck silica gel $60 \mathrm{GF}_{254}$ precoated plates (Darmstadt, Germany) and visualized using a combination of UV254 and UV365 lamps. Silica gel (particle size 200-400 mesh, Marine Chemical Group Co., Qingdao, China) was used for flash chromatography.

\subsection{Synthesis}

4-Nitrophenyl camptothecin-10-yl carbonate (1a): To a stirred solution of 10-hydroxycamptothecin $(3.0 \mathrm{~g}, 8.2 \mathrm{mmol})$ in dry THF $(600 \mathrm{~mL})$ at $0{ }^{\circ} \mathrm{C}$ was added triethylamine $(11.5 \mathrm{~mL}, 82.3 \mathrm{mmol})$ followed by 4-nitrophenyl chloroformate $(6.6 \mathrm{~g}, 32.9 \mathrm{mmol})$. The reaction mixture was stirred at room temperature for $1 \mathrm{~h}$ after which the solvents were then removed under vacuum to give a brownish residue that was purified by column chromatography (200 mesh silica gel, EtOAc) to afford a crude product. Compound 1a (3.59 g, 82\% yield) was purified by recrystallization with hexane and dichloromethane. ${ }^{1} \mathrm{H}-\mathrm{NMR}$ (DMSO-d6) $\delta(\mathrm{ppm}): 8.74(\mathrm{~s}, 1 \mathrm{H}), 8.40(\mathrm{~d}, J=9.2 \mathrm{~Hz}, 2 \mathrm{H}), 8.30(\mathrm{~d}$, $J=9.2 \mathrm{~Hz}, 1 \mathrm{H}), 8.20(\mathrm{~d}, J=2.4 \mathrm{~Hz}, 1 \mathrm{H}), 7.97-7.80(\mathrm{~m}, 1 \mathrm{H}), 7.78(\mathrm{~d}, 2 \mathrm{H}, J=9.2 \mathrm{~Hz}), 7.37(\mathrm{~s}, 1 \mathrm{H})$, $6.54(\mathrm{~s}, 1 \mathrm{H}), 5.44(\mathrm{~s}, 2 \mathrm{H}), 5.32(\mathrm{~s}, 2 \mathrm{H}), 1.85-1.92(\mathrm{~m}, 2 \mathrm{H}), 0.89-0.92(\mathrm{~m}, 3 \mathrm{H})$.

$M P E G-\operatorname{Val}(B o c)$ (2a-2c) and $\operatorname{Val}(B o c)-P E G-\operatorname{Val}(B o c)$ (2d-2f): $N$-Boc-L-valine (1.2 g, 5.5 mmol) was dissolved in dry $\mathrm{CH}_{2} \mathrm{Cl}_{2}(15 \mathrm{~mL})$. Next 4-dimethylaminopyridine (DMAP, $\left.76.0 \mathrm{mg}, 0.6 \mathrm{mmol}\right)$ and 1-ethyl-3-(3-dimethylaminopropyl)carbodiimide hydrochloride $(1.2 \mathrm{~g}, 6.0 \mathrm{mmol})$ were added at $0{ }^{\circ} \mathrm{C}$, and the mixture was stirred for $10 \mathrm{~min}$ after which, $\mathrm{mPEG}$ or PEG $(2.5 \mathrm{mmol})$ was added, and the resulting mixture was stirred overnight at room temperature. After the reaction was complete, the mixture was washed with $0.1 \mathrm{~N} \mathrm{HCl}$ and brine, and dried with anhydrous sodium sulfate. Removal of solvent under vacuum gave a residue that was purified by recrystallization from ethyl ether to afford compounds $\mathbf{2 a}-\mathbf{2 f}(85 \%-95 \%)$.

MPEG-Val (3a-3c) and Val-PEG-Val (3d-3f): To stirred solutions of $\mathbf{2 a - 2 f}$ in $\mathrm{CH}_{2} \mathrm{Cl}_{2}(5 \mathrm{~mL})$ was added trifluoroacetic acid (TFA, $4.6 \mathrm{~mL}$ ) dropwise. The mixture were stirred at room temperature for $1 \mathrm{~h}$, eluted with water, $\mathrm{pH}$ adjusted to 8 by adding aq. $\mathrm{NaHCO}_{3}$, and extracted with $\mathrm{CH}_{2} \mathrm{Cl}_{2}$. The organic layers were combined and dried with anhydrous sodium sulfate. Removal of solvent under vacuum and recrystallization with ethyl ether gave compounds $\mathbf{3 a}-\mathbf{3 f}(87 \%-94 \%$ yield). 
MPEG350-Val (3a): ${ }^{1} \mathrm{H}-\mathrm{NMR}$ (DMSO-d6) $\delta(\mathrm{ppm}): 4.18-4.23(\mathrm{~m}, 1 \mathrm{H}), 4.09-4.15(\mathrm{~m}, 1 \mathrm{H}), 3.62-3.41$ (s, mPEG), 3.24 (s, 3H), $3.14(\mathrm{~d}, J=5.6 \mathrm{~Hz}, 1 \mathrm{H}), 1.96(\mathrm{~s}, 2 \mathrm{H}), 1.88-1.82(\mathrm{~m}, 1 \mathrm{H}),(\mathrm{d}, J=6.8 \mathrm{~Hz}, 3 \mathrm{H})$, $0.83(\mathrm{~d}, J=6.8 \mathrm{~Hz}, 3 \mathrm{H})$.

MPEG $2000-V a l$ (3b): ${ }^{1} \mathrm{H}-\mathrm{NMR}$ (DMSO-d $)_{6} \delta(\mathrm{ppm}): 4.18-4.23(\mathrm{~m}, 1 \mathrm{H}), 4.09-4.15(\mathrm{~m}, 1 \mathrm{H}), 3.69-3.41$ (s, mPEG), 3.24 (s, 3H), $3.15(\mathrm{~d}, J=8.0 \mathrm{~Hz}, 1 \mathrm{H}), 2.05(\mathrm{~s}, 2 \mathrm{H}), 1.87-1.82(\mathrm{~m}, 1 \mathrm{H}), 0.88(\mathrm{~d}, J=6.8 \mathrm{~Hz}$, $3 \mathrm{H}), 0.83(\mathrm{~d}, J=6.8 \mathrm{~Hz}, 3 \mathrm{H})$.

MPEG5000-Val (3c): ${ }^{1} \mathrm{H}-\mathrm{NMR}$ (DMSO-d6) $\delta(\mathrm{ppm})$ : 4.18-4.23 (m, 1H), 4.09-4.15 (m, 1H), 3.78-3.36 (s, PEG), 3.24 (s, 3H, CH $3 \mathrm{O}-), 3.15$ (d, $J=5.3 \mathrm{~Hz}, 1 \mathrm{H}), 1.86-1.80(\mathrm{~m}, 1 \mathrm{H}), 0.88$ (d, $J=6.8 \mathrm{~Hz}, 3 \mathrm{H})$, $0.83(\mathrm{~d}, J=6.8 \mathrm{~Hz}, 3 \mathrm{H})$.

Val-PEG $2000-$ Val (3d): ${ }^{1} \mathrm{H}-\mathrm{NMR}$ (DMSO-d6) $\delta$ (ppm): 4.18-4.23 (m, 2H), 4.09-4.14 (m, 2H), 3.69-3.41 (s, PEG), 3.13 (d, J=5.2 Hz, 2H), 1.86-1.81 (m, 2H), 1.76 (s, 4H), 0.87 (d, J=6.8 Hz, 6H), $0.82(\mathrm{~d}, J=6.8 \mathrm{~Hz}, 6 \mathrm{H})$.

Val-PEG 20000- $\operatorname{Val}(\mathbf{3 e}):{ }^{1} \mathrm{H}-\mathrm{NMR}$ (DMSO-d6) $\delta$ (ppm): 4.12-4.21 (m, 4H), 3.70-3.35 (s, PEG), 3.15 (d, $J=4.0 \mathrm{~Hz}, 2 \mathrm{H}), 1.86-1.90(\mathrm{~m}, 6 \mathrm{H}), 0.90$ (d, $J=6.6 \mathrm{~Hz}, 6 \mathrm{H}), 0.85(\mathrm{~d}, J=6.6 \mathrm{~Hz}, 6 \mathrm{H})$.

Val-PEG $300-V a l$ (3f): ${ }^{1} \mathrm{H}-\mathrm{NMR}\left(\mathrm{DMSO}-d_{6}\right) \delta(\mathrm{ppm}): 4.10-4.23(\mathrm{~m}, 4 \mathrm{H}), 3.60(\mathrm{t}, J=4.8 \mathrm{~Hz}, 2 \mathrm{H}), 3.51$ (s, PEG), 3.12 (d, $J=5.6 \mathrm{~Hz}, 2 \mathrm{H}), 1.79-1.86(\mathrm{~m}, 6 \mathrm{H}), 0.87-0.89$ (dd, $J=6.4,4.0 \mathrm{~Hz}, 6 \mathrm{H}), 0.82-0.84$ (d, $J=6.8 \mathrm{~Hz}, 6 \mathrm{H})$.

MPEG-Val-HCPT (4a-4c) and HCPT-Val-PEG-Val-HCPT (4d-4f): Compound 1a (0.6 g, $1.1 \mathrm{mmol})$ was dissolved in anhydrous DMF $(3 \mathrm{~mL})$ and $\mathrm{CH}_{2} \mathrm{Cl}_{2}(3 \mathrm{~mL})$. Next, 3a-3f $(0.7 \mathrm{mmol})$ were added to make individual mixtures that were then stirred at room temperature. After reacting for $1 \mathrm{~h}$, the solutions were washed with brine and the organic layers were combined and dried with anhydrous sodium sulfate. Removal of solvent under vacuum and column chromatography (200 mesh silica gel) with $\mathrm{CH}_{2} \mathrm{Cl}_{2} / \mathrm{CH}_{3} \mathrm{OH}(30: 1, \mathrm{v} / \mathrm{v})$ as eluent gave the crude products. Compounds $4 \mathbf{a}-\mathbf{4 f}(63 \%-67 \%$ yield) were obtained by recrystallization from ethyl ether.

MPEG $350-V a l-H C P T$ (4a): ${ }^{1} \mathrm{H}-N M R\left(D M S O-d_{6}\right) \delta(\mathrm{ppm}): 8.68(\mathrm{~s}, 1 \mathrm{H}), 8.43(\mathrm{~d}, J=8.0 \mathrm{~Hz}, 1 \mathrm{H}), 8.18$ $(\mathrm{d}, J=9.2 \mathrm{~Hz}, 1 \mathrm{H}), 7.92(\mathrm{~d}, J=2.0 \mathrm{~Hz}, 1 \mathrm{H}), 7.63-7.66(\mathrm{dd}, J=9.2,2.4 \mathrm{~Hz}, 1 \mathrm{H}), 7.34(\mathrm{~s}, 1 \mathrm{H}), 6.52$ (s, $1 \mathrm{H}), 5.43(\mathrm{~s}, 2 \mathrm{H}), 5.30(\mathrm{~s}, 2 \mathrm{H}), 4.28-4.33(\mathrm{~m}, 1 \mathrm{H}), 4.18-4.22(\mathrm{~m}, 1 \mathrm{H}), 4.03-4.07(\mathrm{~m}, 1 \mathrm{H}), 3.49-3.66$ (s, mPEG), $3.23(\mathrm{~s}, 3 \mathrm{H}), 2.14-2.17(\mathrm{~m}, 1 \mathrm{H}), 1.84-1.91(\mathrm{~m}, 2 \mathrm{H}), 0.98-1.01(\mathrm{~m}, 6 \mathrm{H}), 0.89$ (t, $J=7.2 \mathrm{~Hz}, 3 \mathrm{H})$.

MPEG $2000-V a l-H C P T(\mathbf{4 b}):{ }^{1} \mathrm{H}-\mathrm{NMR}$ (DMSO-d6) $\delta(\mathrm{ppm}): 8.68(\mathrm{~s}, 1 \mathrm{H}), 8.44(\mathrm{~d}, J=8.4 \mathrm{~Hz}, 1 \mathrm{H}), 8.19$ $(\mathrm{d}, J=9.2 \mathrm{~Hz}, 1 \mathrm{H}), 7.92(\mathrm{~d}, J=2.4 \mathrm{~Hz}, 1 \mathrm{H}), 7.63-7.66(\mathrm{dd}, J=9.2,2.8 \mathrm{~Hz}, 1 \mathrm{H}), 7.35(\mathrm{~s}, 1 \mathrm{H}), 6.53$ (s, $1 \mathrm{H}), 5.43(\mathrm{~s}, 2 \mathrm{H}), 5.30(\mathrm{~s}, 2 \mathrm{H}), 4.28-4.32(\mathrm{~m}, 1 \mathrm{H}), 4.17-4.21(\mathrm{~m}, 1 \mathrm{H}), 4.02-4.06(\mathrm{~m}, 1 \mathrm{H}), 3.41-3.68$ (s, mPEG), $3.24(\mathrm{~s}, 3 \mathrm{H}), 2.13-2.18(\mathrm{~m}, 1 \mathrm{H}), 1.84-1.91(\mathrm{~m}, 2 \mathrm{H}), 0.98-1.01(\mathrm{~m}, 6 \mathrm{H}), 0.89(\mathrm{t}$, $J=7.2 \mathrm{~Hz}, 3 \mathrm{H})$. 
MPEG5000-Val-HCPT (4c): ${ }^{1} \mathrm{H}-\mathrm{NMR}$ (DMSO-d6) $\delta(\mathrm{ppm}): 8.69(\mathrm{~s}, 1 \mathrm{H}), 8.44(\mathrm{~d}, J=8.0 \mathrm{~Hz}, 1 \mathrm{H}), 8.19$ $(\mathrm{d}, J=8.8 \mathrm{~Hz}, 1 \mathrm{H}), 7.91(\mathrm{~s}, 1 \mathrm{H}), 7.65(\mathrm{~d}, J=7.6 \mathrm{~Hz}, 1 \mathrm{H}), 7.35(\mathrm{~s}, 1 \mathrm{H}), 6.54(\mathrm{~s}, 1 \mathrm{H}), 5.43(\mathrm{~s}, 2 \mathrm{H}), 5.30$ (s, 2H), 4.29-4.32 (m, 1H), 4.17-4.20 (m, 1H), 4.03-4.06 (m, 1H), 3.44-3.68 (s, mPEG), 3.24 (s, 3H), 2.14-2.18 (m, 1H), 1.86-1.89 (m, 2H), 0.98-1.01 (m, 6H), 0.89 (t, J=7.2 Hz, 3H).

HCPT-Val-PEG $2000-V a l-H C P T$ (4d): ${ }^{1} \mathrm{H}-\mathrm{NMR}$ (DMSO-d 6 ) $\delta(\mathrm{ppm}): 8.68$ (s, 2H), 8.43 (d, $J=8.0 \mathrm{~Hz}$, $2 \mathrm{H}), 8.19(\mathrm{~d}, J=9.2 \mathrm{~Hz}, 2 \mathrm{H}), 7.91(\mathrm{~d}, J=2.4 \mathrm{~Hz}, 2 \mathrm{H}), 7.63-7.66(\mathrm{dd}, J=9.2,2.4 \mathrm{~Hz}, 2 \mathrm{H}), 7.35$ (s, $2 \mathrm{H}), 6.53(\mathrm{~s}, 2 \mathrm{H}), 5.43(\mathrm{~s}, 4 \mathrm{H}), 5.30(\mathrm{~s}, 4 \mathrm{H}), 4.28-4.32(\mathrm{~m}, 2 \mathrm{H}), 4.17-4.20(\mathrm{~m}, 2 \mathrm{H}), 4.02-4.06$ (m, 2H), 3.45-3.66 (s, PEG), 2.13-2.18 (m, 2H), 1.84-1.91 (m, 4H), 0.98-1.01 (m,12H), 0.89 (t, $J=7.2 \mathrm{~Hz}, 6 \mathrm{H})$.

HCPT-Val-PEG $20000-V a l-H C P T$ (4e): ${ }^{1} \mathrm{H}-\mathrm{NMR}$ (DMSO-d6) $\delta(\mathrm{ppm}): 8.70$ (s, 2H), 8.45 (d, J=7.2 Hz, 2H), $8.20(\mathrm{~d}, J=8.8 \mathrm{~Hz}, 2 \mathrm{H}), 7.93(\mathrm{~s}, 2 \mathrm{H}), 7.65-7.67(\mathrm{~d}, J=7.6,2 \mathrm{H}), 7.37(\mathrm{~s}, 2 \mathrm{H}), 6.55$ (s, 2H), 5.44 (s, 4H), 5.32 (s, 4H), 4.21-4.31 (m, 4H), 4.06-4.08 (m, 2H), 3.52-3.69 (s, PEG), 2.17-2.18 (m, 2H), $1.89(\mathrm{~m}, 4 \mathrm{H}), 1.01(\mathrm{~m}, 12 \mathrm{H}), 0.83(\mathrm{t}, J=7.2 \mathrm{~Hz}, 6 \mathrm{H})$.

HCPT-Val-PEG300-Val-HCPT (4f): ${ }^{1} \mathrm{H}-\mathrm{NMR}$ (DMSO-d6) $\delta(\mathrm{ppm}): 8.66$ (s, 2H), 8.44 (d, J=8.0 Hz, 2H), $8.17(\mathrm{~d}, J=8.8 \mathrm{~Hz}, 2 \mathrm{H}), 7.90(\mathrm{~s}, 2 \mathrm{H}), 7.63(\mathrm{~d}, J=8.4,2 \mathrm{H}), 7.34(\mathrm{~s}, 2 \mathrm{H}), 6.53$ (s, 2H), 5.43 (s, $4 \mathrm{H}), 5.28(\mathrm{~s}, 4 \mathrm{H}), 4.18-4.33(\mathrm{~m}, 4 \mathrm{H}), 4.03-4.07(\mathrm{~m}, 2 \mathrm{H}), 3.50-3.65$ (s, PEG), 2.16-2.17 (m, 2H), $1.85-1.91(\mathrm{~m}, 4 \mathrm{H}), 0.98-1.00(\mathrm{~m}, 12 \mathrm{H}), 0.89(\mathrm{t}, J=7.2 \mathrm{~Hz}, 6 \mathrm{H})$.

$M P E G_{2000}$ (4-nitrophenyl) carbonate (1b): Methoxypolyethylene glycol 2000 (mPEG $2000,3.0 \mathrm{~g}$, $1.5 \mathrm{mmol})$ was dissolved in anhydrous $\mathrm{CH}_{2} \mathrm{Cl}_{2}(15 \mathrm{~mL})$. Triethylamine $(4.2 \mathrm{~mL}, 30.0 \mathrm{mmol})$ and 4-nitrophenyl chloroformate $(1.2 \mathrm{~g}, 6.0 \mathrm{mmol})$ were added at $0{ }^{\circ} \mathrm{C}$ and the mixture was stirred at room temperature for $3 \mathrm{~h}$. The mixture was concentrated under vacuum, purified by column chromatography $\left(\mathrm{CH}_{2} \mathrm{Cl}_{2} / \mathrm{CH}_{3} \mathrm{OH}, 25: 1, \mathrm{v} / \mathrm{v}\right)$ and recrystallized from ethyl ether to afford compound $\mathbf{1 b}(2.6 \mathrm{~g}, 80 \%$ yield). ${ }^{1} \mathrm{H}-\mathrm{NMR}\left(\mathrm{DMSO}-d_{6}\right) \delta(\mathrm{ppm}): 8.32(\mathrm{~d}, J=9.2 \mathrm{~Hz}, 2 \mathrm{H}), 7.57(\mathrm{~d}, J=9.2 \mathrm{~Hz}, 2 \mathrm{H}), 4.38-4.36$ (m, 2H), 3.71-3.42 (s, mPEG), 3.24 (s, 3H).

mPEG $2000-O C O-H C P T$ (5): To a stirred solution of HCPT (150.0 mg, $0.4 \mathrm{mmol})$ in anhydrous DMF were added triethylamine $(0.24 \mathrm{~mL}, 1.7 \mathrm{mmol})$ and compound $\mathbf{1 b}(0.6 \mathrm{~g}, 0.3 \mathrm{mmol})$. The reaction mixture was stirred at $60{ }^{\circ} \mathrm{C}$ for $45 \mathrm{~min}$, diluted with water, and extracted with $\mathrm{CH}_{2} \mathrm{Cl}_{2}$. The organic layer was combined, dried with anhydrous sodium sulfate, concentrated under vacuum, purified by column chromatography $\left(\mathrm{CH}_{2} \mathrm{Cl}_{2} / \mathrm{CH}_{3} \mathrm{OH}, 25: 1, \mathrm{v} / \mathrm{v}\right)$ and recrystallized with ethyl ether to give compound 5 (350.0 g, 55\% yield). ${ }^{1} \mathrm{H}-\mathrm{NMR}\left(\mathrm{DMSO}-d_{6}\right) \delta(\mathrm{ppm}): 8.70(\mathrm{~s}, 1 \mathrm{H}), 8.24(\mathrm{~d}, J=9.2 \mathrm{~Hz}, 1 \mathrm{H})$, $8.05(\mathrm{~d}, J=2.4 \mathrm{~Hz}, 1 \mathrm{H}), 7.78-7.81(\mathrm{dd}, J=9.2,2.4 \mathrm{~Hz}, 2 \mathrm{H}), 7.36(\mathrm{~s}, 1 \mathrm{H}), 6.55(\mathrm{~s}, 1 \mathrm{H}), 5.44(\mathrm{~s}, 2 \mathrm{H})$, $5.31(\mathrm{~s}, 2 \mathrm{H}), 4.39$ (t, 2H), 3.75-3.41 (s, mPEG), 3.24 (s, 3H), 1.82-1.93 (m, 2H), 0.89 (t, J=7.2 Hz, 3H).

MPEG 2000 4-methylbenzenesulfonate (7): $\mathrm{mPEG}_{2000}(3.0 \mathrm{~g}, 1.5 \mathrm{mmol})$ was dissolved in anhydrous $\mathrm{CH}_{2} \mathrm{Cl}_{2}(15.0 \mathrm{~mL})$. Triethylamine $(4.2 \mathrm{~mL}, 29.8 \mathrm{mmol})$ and 4-methoxybenzenesulfonyl chloride $(0.9 \mathrm{~g}$, $5.1 \mathrm{mmol}$ ) were added at $0{ }^{\circ} \mathrm{C}$. The mixture was heated to reflux for $5 \mathrm{~h}$, diluted with $\mathrm{CH}_{2} \mathrm{Cl}_{2}$, and washed with $1 \mathrm{~N} \mathrm{HCl}$ and brine. The organic layer was dried with anhydrous sodium sulfate, concentrated under vacuum and purified by recrystallization with ethyl ether to give compound 7 
(3.0 g, 91\% yield). ${ }^{1} \mathrm{H}-\mathrm{NMR}$ (DMSO- $\left.d_{6}\right) \delta(\mathrm{ppm}): 7.78(\mathrm{~d}, J=8.4 \mathrm{~Hz}, 2 \mathrm{H}), 7.48(\mathrm{~d}, J=8.0 \mathrm{~Hz}, 2 \mathrm{H})$, $4.11(\mathrm{t}, J=4.4 \mathrm{~Hz}, 2 \mathrm{H}), 3.69-3.45$ (s, mPEG), 3.24 (s, 3H), 2.42 (s, 3H).

1-Bromo mPEG $2000(8)$ : To a solution of compound $7(3.0 \mathrm{~g}, 1.4 \mathrm{mmol})$ in dry acetone $(15.0 \mathrm{~mL})$ was added $\mathrm{LiBr}(0.6 \mathrm{~g}, 7.0 \mathrm{mmol})$. The mixture was refluxed overnight, concentrated under vacuum, purified by column chromatography (200 mesh silica gel, $\mathrm{CH}_{2} \mathrm{Cl}_{2} / \mathrm{CH}_{3} \mathrm{OH}, 25: 1$, v/v), and recrystallized with ethyl ether to give compound 8 (2.3 g, 80\% yield). ${ }^{1} \mathrm{H}-\mathrm{NMR}$ (DMSO- $\left.d 6\right) \delta$ (ppm): 3.80-3.77 (t, $J=5.8 \mathrm{~Hz}, 2 \mathrm{H}), 3.75-3.73(\mathrm{t}, J=4.8 \mathrm{~Hz}, 2 \mathrm{H}), 3.69-3.45$ (s, mPEG), 3.30 (s, 3H, $\mathrm{CH}_{3} \mathrm{O}-$ ).

mPEG $2000-O-H C P T(6)$ : To a solution of HCPT $(350.0 \mathrm{mg}, 1.0 \mathrm{mmol})$ and potassium carbonate (270.0 $\mathrm{mg}, 2.0 \mathrm{mmol})$ in dry DMSO $(2.5 \mathrm{~mL})$ was added compound $\mathbf{8}(1.0 \mathrm{~g}, 0.5 \mathrm{mmol})$. The mixture was allowed to react for $3 \mathrm{~h}$ at $60{ }^{\circ} \mathrm{C}$ and the reaction mixture was directly loaded onto a silica gel column. Purification of the reaction mixture by column chromatography on silica gel using $4 \%$ $\mathrm{CH}_{3} \mathrm{OH}$ in $\mathrm{CH}_{2} \mathrm{Cl}_{2}$ as an eluent gave compound 6 (650.0 mg, 57\% yield). ${ }^{1} \mathrm{H}-\mathrm{NMR}$ (DMSO-d6) $\delta$ (ppm): $8.52(\mathrm{~s}, 1 \mathrm{H}), 8.06(\mathrm{~d}, J=9.2 \mathrm{~Hz}, 1 \mathrm{H}), 7.53(\mathrm{~s}, 1 \mathrm{H}), 7.51(\mathrm{~d}, J=2.8 \mathrm{~Hz}, 1 \mathrm{H}) 7.28(\mathrm{~s}, 1 \mathrm{H}), 6.50$ (s, 1H), $5.42(\mathrm{~s}, 2 \mathrm{H}), 5.26(\mathrm{~s}, 2 \mathrm{H}), 4.29(\mathrm{t}, J=4.2 \mathrm{~Hz}, 2 \mathrm{H}), 3.85(\mathrm{t}, J=4.2 \mathrm{~Hz}, 2 \mathrm{H}), 3.41-3.68$ (s, mPEG),3.24 (s, 3H), 1.83-1.90 (m, 2H), 0.89 (t, $J=7.2 \mathrm{~Hz}, 3 \mathrm{H})$.

\subsection{Cytotoxicity Study}

The cytotoxicity of the target compounds were evaluated by the MTT assay. Cells were seeded at a density of $5 \times 10^{4}$ cells $/ \mathrm{mL}$ in 96 -well microplates ( $100 \mu \mathrm{L} /$ well). After $2 \mathrm{~h}$ for K562 cells and $24 \mathrm{~h}$ for HepG2 and HT-29 cells, media containing tested compounds were added in triplicate. After a $48 \mathrm{~h}$ incubation, the media were replaced with PBS medium containing $0.5 \mathrm{mg} / \mathrm{mL}$ MTT and the cells were incubated for another $4 \mathrm{~h}$. Next, the medium was removed and $100 \mu \mathrm{L}$ DMSO was added to each well to dissolve the formazan. The absorbance at $570 / 630 \mathrm{~nm}$ was measured for K562 cells and the absorbance at 490/630 nm was measured for HepG2 and HT-29 cells using a Thermo (Waltham, MA, USA) microplate reader. The untreated controls were considered as having a cell viability value of $100 \%$. The half-maximal inhibiting concentration $\left(\mathrm{IC}_{50}\right)$ values were obtained by nonlinear regression using GraphPad Prism 5.0. IC 50 was measured three times for each compound.

\subsection{Stability Test}

The stability of all the compounds in PBS was examined by HPLC using a C18 analytical column. The mobile phase was composed of water and acetonitrile at a ratio of 60:40. The amount of the compound and its metabolite HCPT were flowed at a rate of $1.0 \mathrm{~mL} \cdot \mathrm{min}^{-1}$ and detected by a UV wavelength of $360 \mathrm{~nm}$. Stability test in PBS: each compound was dissolved in PBS (pH 7.4), incubated at $37^{\circ} \mathrm{C}$ and analyzed at $1,2,3$, and $4 \mathrm{~h}$.

\subsection{Statistics}

Values are $\mathrm{IC}_{50}(\mu \mathrm{M})$ means \pm SEM of three separate experiments. All statistical analysis was performed by using GraphPad Prism software version 5.0 (GraphPad Software, Inc., San Diego, CA, USA). ${ }^{*} p<0.05 ; * * p<0.01$, compared with the control group (HCPT). 


\section{Conclusions}

In order to improve the pharmacokinetic properties of HCPT and find new water soluble camptothecin type drugs with higher potency and lower side effects, eight novel conjugates of HCPT were designed and synthesized using different lengths of PEG as carriers and three types of linkages. The rate of release of HCPT and antitumor activity of the obtained conjugates are related to the length of the PEG chain and the linker type. Our results showed that conjugates with relatively short PEG chains and valine-spacers exhibited excellent antitumor activity and controlled release of HCPT. Further exploration of the mechanism on the influence of PEG length and different spacers on the HCPT release properties and antitumor activity is ongoing in our lab.

\section{Supplementary Materials}

Supplementary materials can be accessed at: http://www.mdpi.com/1420-3049/20/05/9393/s1.

\section{Acknowledgments}

This work was supported by the National Natural Science Foundation of China (No. 81302649), the International Science \& Technology Cooperation Program of China (2013DFA31160), Tianjin Municipal Science and Technology Commission (14JCQNJC13200) and the Ministry of Education Changjiang Scholars and Innovative Research Team Development Plan (IRT1166).

\section{Author Contributions}

N.G. and P.Y. conceived the project and participated in all steps of the research, interpreted the results and wrote the paper. N.G., D.J. and X.Y. executed the chemical synthesis and the stability test. L.W. and Y.-O.T. performed the biological assays. All authors have seen and commented on the manuscript.

\section{Conflicts of Interest}

The authors declare no conflict of interest.

\section{References}

1. Ohwada, J.; Ozawa, S.; Kohchi, M. Synthesis and biological activities of a pH-dependently activated water-soluble prodrug of a novel hexacyclic camptothecin analog. Bioorg. Med. Chem. Lett. 2009, 19, 2772-2776.

2. Basili, S.; Moro, S. Novel camptothecin derivatives as topoisomerase I inhibitors. Expert Opin. Ther. Patents. 2009, 19, 555-574.

3. Wang, J.Q.; Sun, X.R.; Mao, W.W.; Sun, W.L.; Tang, J.B.; Sui, M.H.; Shen, Y.Q.; Gu, Z.W. Tumor redox heterogeneity-responsive prodrug nanocapsules for cancer chemotherapy. Adv. Mater. 2013, 25, 3670-3676. 
4. Shamay, Y.; Paulin, D.; Ashkenasy, G.; David, A. E-selectin binding peptide-polymer-drug conjugates and their selective cytotoxicity against vascular endothelial cells. Biomaterials 2009, $30,6460-6468$.

5. Xu, Z.G.; Wang, D.D.; Xu, S.; Liu, X.Y.; Zhang, X.Y.; Zhang, H.X. Preparation of camptothecin prodrug with glutathione-responsive disulfide linker for anticancer drug delivery. Chem. Asian J. 2014, 9, 199-205.

6. Lee, M.H.; Kim, J.Y.; Han, J.H.; Bhuniya, S.; Sessler, J.L.; Kang, C.; Kim, J.S. Direct fluorescence monitoring of the delivery and cellular uptake of a cancer-targeted RGD peptide-appended naphthalimide theragnostic prodrug. J. Am. Chem. Soc. 2012, 134, 12668-12674.

7. Conover, C.D.; Greenwald, R.B.; Pendri, A.; Shum, K.L. Camptothecin delivery systems: The utility of amino acid spacers for the conjugation of camptothecin with polyethylene glycol to create prodrugs. Anti-Cancer Drug Des. 1999, 14, 499-506.

8. Fleming, A.B.; Haverstick, K.; Saltzman, W.M. In vitro cytotoxicity and in vivo distribution after direct delivery of PEG-camptothecin conjugates to the rat brain. Bioconjugate Chem. 2004, 15, 1364-1375.

9. Senter, P.D.; Beam, K.S.; Mixan, B.; Wahl, A.F. Identification and activities of human carboxylesterases for the activation of CPT-11, a clinically approved anticancer drug. Bioconjugate Chem. 2001, 12, 1074-1080.

10. De Jong, F.A.; Mathijssen, R.H.J.; Xie, R.; Verweij, J.; Sparreboom, A. Flat-fixed dosing of irinotecan: Influence on pharmacokinentic and pharmacodynamics variability. Clin. Cancer Res. 2004, 10, 4068-4071.

11. Endo, M.; Ohwada, J.; Ogawa, K.; Yamada-Okabe, H. A water soluble prodrug of a novel camptothecin analog is efficacious against breast cancer resistance protein-expressing tumor xenografts. Cancer Chemother. Pharmacol. 2010, 65, 363-371.

12. Lv, J.; Guo, N.; Wen, S.P.; Teng, Y.O.; Ma, M.X.; Yu, P. Synthesis and antitumor activity evaluation of a novel series of camptothecin analogs. J. Asian Nat. Prod. Res. 2013, 15, 863-874.

Sample Availability: Samples of the compounds $\mathbf{4 a - 4 f}$ are available from the authors.

(C) 2015 by the authors; licensee MDPI, Basel, Switzerland. This article is an open access article distributed under the terms and conditions of the Creative Commons Attribution license (http://creativecommons.org/licenses/by/4.0/). 